\title{
Green Functions in the Theory of Local Unrenormalizable Interactions
}

\author{
M. K. VOLKOV \\ Joint Institute for Nuclear Research, Dubna, USSR
}

Received July 9, 1969

\begin{abstract}
The method suggested earlier for the description of unrenormalizable interactions of massless particles [1-4] is generalized to the case of interactions of particles with non-zero rest mass.
\end{abstract}

\section{Introduction}

In a number of previous papers [1-4] the problem of the construction of the Green functions in the momentum space in theories describing unrenormalizable interactions was investigated. In the $x$-space these functions have an essential singularity when their argument is zero. Therefore the construction of the Fourier transforms of these functions is a non-trivial problem.

In the case when the rest masses of particles are zero the problem conserve all its characteristic features while the calculations become essentially, simpler. This just explains the fact that in previous papers one investigated the interactions of massless particles. The attention was focussed on the main problem, the transition from the Green functions constructed in the $\mathrm{x}$-space to those in the momentum space. A method has been developed which allows one to perform such a transformation. It may be used in theories which describe a wide class of unrenormalizable interactions. The Green functions constructed by this method satisfy the conditions of causality and unitary of the S-matrix.

However, to complete the investigations performed it is necessary to generalize the methods developed for the case of unrenormalizable interactions of massless particles to the general case of unrenormalizable interaction of particles with arbitrary rest mass. This paper is just devoted to this problem.

A two-point Green function which depends exponentially in the $\mathrm{x}$ space on the propagator of a free scalar particle with rest mass $m$ is investigated. Such functions are often found in theories describing local unrenormalizable interactions [1-8]. The calculations were carried 
out using an approximate expression for the free scalar particle propagator. The meaning of this approximation is cleared up further by the example of the absorptive part of the two-point Green function. The method suggested here for the construction of the Green functions in the momentum space is in complete agreement with the conditions of locality and unitarity of the S-matrix.

The last section is devoted to the generalization of the method developed to the case of more general unrenormalizable interactions.

In the limit of high energies the results obtained here are in complete agreement with those obtained earlier for the case of interaction of massless particles $[1-4]$.

\section{Two-Point Green Function}

Applying perturbation theory for the description of unrenormalizable elementary particle interactions like e.g. pion-nucleon interaction of neutral particles with pseudo-vector coupling $[2,4,5]$ or parity nonconserving interactions of a neutral meson with nucleons [8] we are led to a two-point Green function of the type

$$
\tilde{F}_{i}(x)=\Psi_{i}(x) \exp \left\{-i g^{2} \Delta(x)\right\},
$$

where $\mathrm{g}$ is the coupling constant, $\Delta(x)$ is the propagator of a free scalar particle, $\Psi_{i}(x)$ stands for a function of the free spinor particle propagator. For the sake of simplicity we consider the case $\Psi_{0}(x)=1$. The generalization to the case $\Psi_{1}(x)=S(x)$ or $\Psi_{2}(x)=S p\{S(x) S(-x)\}$, where $S(x)$ is the propagator of a free spinor particle, is not difficult [2].

To construct the Fourier transform of the function $\tilde{F}_{0}(x)$ we introduce a subsidiary function $\tilde{\Phi}_{0}(x)$ differing from $\tilde{F}_{0}(x)$ by the sign in the exponential. In the unphysical region $\mathrm{p}^{2}<0$ the Fourier transform of this function will be constructed in a certain approximation. This approximation well describes the exact function at large momenta. Then a transition will be performed to the desired function by analytical continuation with respect to the coupling constant in the same manner as it was done in Ref. [2] for the case of massless scalar particles. form

The Fourier transform of the function $\tilde{\Phi}_{0}(\mathrm{x})$ can be written in the

$$
\Phi_{0}(p)=i(2 \pi)^{4} \delta^{(4)}(p)+\frac{(2 \pi)^{2} \chi}{p^{2}-m^{2}+i \varepsilon}+\varphi_{\varkappa}(p)
$$

where

$$
\varphi_{\varkappa}(p)=i \int d^{4} x e^{i p x}\left\{\exp \left[i(2 \pi)^{2} \varkappa \Delta(x)\right]-1-i(2 \pi)^{2} \varkappa \Delta(x)\right\}
$$


and $x=\left(\frac{\mathrm{g}}{2 \pi}\right)^{2}$. In the unphysical region $\mathrm{p}^{2}<0$ we choose the coordinate system in which $p=\{0, \mathbf{p}\}$. In this co-ordinate system the contour of integration over the time variable in (3) can be rotated at angle $-\frac{\pi}{2}$. As a result, we arrive at the Euclidean metric. By choosing the polar co-ordinates and integrating over the angular variables we get for the function $\varphi_{\varkappa}(p)$ the following equation

$\varphi_{\varkappa}(p)=(2 \pi \varkappa)^{2} \frac{m}{|p|} \int_{0}^{1} d t(1-t) \int_{0}^{\infty} d r J_{1}\left(\frac{|p|}{m} r\right)\left[K_{1}(r)\right]^{2} \exp \left\{-t \varkappa m^{2} \frac{K_{1}(r)}{r}\right\}$

where $|p|=\sqrt{-p^{2}}=\sqrt{p^{2}}$ and $J_{1}(z), K_{1}(z)$ are Bessel function. For various kinds of Bessel functions we shall use here and in what follows the notations of Watson [9].

It is doubtful that the integral (4) can be taken exactly. However it can be rather well estimated replacing the $K_{1}(r)$ function by an exponential divided by $r$

$$
K_{1}(r) \sim \frac{e^{-r}}{r}
$$

For very small and large values of the argument this function well approximate the $\mathrm{K}_{1}(\mathrm{r})$ function. Therefore with such a replacement one may expect to obtain a result which rather well reproduces the behaviour of the true function $\varphi_{\chi}(\mathrm{p})$.

\section{Qualitative Estimates of the Green Function}

Before to proceed to the calculation of the integral (4) in the approximation indicated it is useful to consider more rough approximation. It consists in that in the exponential the $\frac{K_{1}(r)}{r}$ function is replaced by the function corresponding to the propagator of a scalar massless particle, and the square of the $K_{1}(r)$ function before the exponential is replaced as is shown in (5)

$$
\overline{\bar{\varphi}}_{\varkappa}(p)=(2 \pi \varkappa)^{2} \frac{m}{|p|} \int_{0}^{1} d t(1-t) \int_{0}^{\infty} \frac{d r}{r^{2}} e^{-2 r} J_{1}\left(\frac{|p|}{m} r\right) \exp \left\{-t \frac{\varkappa m^{2}}{r^{2}}\right\} .
$$


In what follows we mark by double bars the functions calculated in this approximation and by a single bar the functions calculated in the approximation (5). We need the approximation (6) in order to demonstrate by more simple example the technique of calculation of the integrals of the type (6) and the analytic continuation of the function obtained with respect to the variable $\mathrm{p}^{2}$ from the region $\mathrm{p}^{2}<0$ in which Eq. (6) holds, to the region $0 \leqq \mathrm{p}^{2} \leqq 4 \mathrm{~m}^{2}$ as well as to the physical region $\mathrm{p}^{2}>4 \mathrm{~m}^{2}$. Further these operations will ve extended to the approximation (5).

In the integrand of Eq. (6) the exponential can be represented in the form of the Mellin-Barnes integral [10]

$$
\exp \left\{-t \frac{x m^{2}}{r^{2}}\right\}=\frac{1}{2 i} \int_{-\alpha+i \infty}^{-\alpha-i \infty} d z \frac{\left(t x m^{2}\right)^{z} r^{-2 z}}{\sin \pi z \Gamma(z+1)}
$$

where $0<\alpha<1, \Gamma(\mathrm{z}+1)$ is the gamma function. We insert (7) into (6). Then it is easy to make oneself sure that all the integrals converge absolutely and therefore it is possible to alter the order of integration. Integrating over the variable $t$ and changing the order of integration over $\mathrm{r}$ and $\mathrm{z}$ we rewrite (6) as follows:

$$
\overline{\bar{\varphi}}_{\varkappa}(p)=(2 \pi \varkappa)^{2} \frac{m}{|p|} \frac{1}{2 i} \int_{-\alpha+i \infty}^{-\alpha-i \infty} d z \frac{\left(\varkappa m^{2}\right)^{z}}{\sin \pi z \Gamma(z+3)} \int_{0}^{\infty} d r r^{-2(z+1)} e^{-2 r} J_{1}\left(\frac{|p|}{m} r\right) .
$$

The integration over $r$ leads to the appearance of a spherical function $P_{-2(z+1)}^{-1}\left[\left(1+\frac{p^{2}}{4 m^{2}}\right)^{-\frac{1}{2}}\right]($ see Ref. [11]) $\overline{\bar{\varphi}}_{\chi}(p)=i \pi^{3} \chi^{2} \sqrt{1-\frac{4 m^{2}}{p^{2}}} \int_{-\alpha+i \infty}^{-\alpha-i \infty} d z \frac{\left[\varkappa\left(4 m^{2}-p^{2}\right)\right]^{z}}{(\sin \pi z)^{2} \cos \pi z \Gamma(z+3) \Gamma(2 z+1)}$

$$
\cdot P_{-2(z+1)}^{-1}\left[\left(1-\frac{p^{2}}{4 m^{2}}\right)^{-\frac{1}{2}}\right]
$$

Using for $P_{-2(z+1)}^{-1}(a)$ the representation $[10,11]$

$$
P_{-2(z+1)}^{-1}(a)=\left(\frac{2}{1+a}\right)^{2(z+1)}\left(\frac{1-a}{1+a}\right)^{\frac{1}{2}} F\left(2 z+2,2 z+3 ; 2 ; \frac{a-1}{a+1}\right),
$$


where $F\left(2 z+2,2 z+3 ; 2 ; \frac{a-1}{a+1}\right)$ is the hypergeometric function, it is easy to see that the function $P_{-2(z+1)}^{-1}(a)$ has no singularities in the right half-plane $\operatorname{Re} z>0$ and no zeros on the real positive axis. Therefore, the integral over $z$ is easily taken and the function $\overline{\bar{\varphi}}_{x}(p)$ is represented in the form of a series. We do not write out here this series in an explicit form, we show only how to derive from the function $\overline{\bar{\varphi}}_{x}(p)$ the function $\bar{f}(p)$. This function is connected with the approximate expression for the Fourier transform of the desired function by the relation

$$
\overline{\bar{F}}(p)=i(2 \pi)^{4} \delta^{(4)}(p)-\frac{(2 \pi)^{2} x}{p^{2}-m^{2}+i \varepsilon}+\overline{\bar{f}}(p) .
$$

Following Ref. [2] $\overline{\bar{f}}(p)$ can be expressed in terms of $\overline{\bar{\varphi}}_{x}(p)$ by means of the formula

$$
\overline{\bar{f}}(p)=\frac{1}{2}\left\{\overline{\bar{\varphi}}_{x e^{\imath \pi}}(p)+\overline{\bar{\varphi}}_{x e^{-\imath \pi}}(p)\right\} .
$$

Inserting (9) into (12) and integrating over $\mathrm{z}$ we get

$$
\begin{aligned}
\overline{\bar{f}}(p)=-2(\pi x)^{2} \sqrt{1-\frac{4 m^{2}}{p^{2}}} \sum_{n=0}^{\infty} \frac{\partial}{\partial v} \\
\left.\cdot\left\{\frac{\left[x\left(4 m^{2}-p^{2}\right)\right]^{v}}{\Gamma(v+3) \Gamma(2 v+1)} P_{-2(v+1)}^{-1}\left[\left(1-\frac{p^{2}}{4 m^{2}}\right)^{-\frac{1}{2}}\right]\right\}\right|_{v=n} .
\end{aligned}
$$

The formula (13) is valid for the $\mathrm{p}^{2}$-values lying in the unphysical domain $\mathrm{p}^{2}<0$.

In order to continue analytically $\overline{\bar{f}}(p)$ into to the next domain $0 \leqq p^{2} \leqq 4 m^{2}$ it is convenient to return to the function $\overline{\bar{\varphi}}_{x}(p)$ written in the form of (8). In the region we are interested in it takes on the form

$$
\overline{\bar{\varphi}}_{\varkappa}(p)=(2 \pi \varkappa)^{2} \frac{m}{p} \frac{1}{2 i} \int_{-\alpha+i \infty}^{-\alpha-i \infty} d z \frac{\left(\varkappa m^{2}\right)^{z}}{\sin \pi z \Gamma(z+3)} \int_{0}^{\infty} d r r^{-2(z+1)} e^{-2 r} I_{1}\left(\frac{p}{m} r\right),
$$

where $p=\sqrt{p^{2}}, I_{1}\left(\frac{p}{m} r\right)$ is the Bessel function of the imaginary argument. The integration over $\mathrm{r}$ leads again to the appearance of the spherical function $\mathscr{P}_{-2(z+1)}^{-1}\left[\left(1-\frac{p^{2}}{4 m^{2}}\right)^{-\frac{1}{2}}\right]$ which in the domain $\mathrm{p}^{2}<0$ is 
connected with the former by the relation $[10,11]$

$$
\begin{aligned}
& P_{-2(z+1)}^{-1}\left[\left(1-\frac{p^{2}}{4 m^{2}}\right)^{-\frac{1}{2}}\right] \\
& =\frac{i}{2}\left\{\mathscr{P}_{-2(z+1)}^{-1}\left[\left(1-\frac{p^{2}}{4 m^{2}}\right)^{-\frac{1}{2}}-i 0\right]-\mathscr{P}_{-2(z+1)}^{-1}\left[\left(1-\frac{p^{2}}{4 m^{2}}\right)^{-\frac{1}{2}}+i 0\right]\right\} .
\end{aligned}
$$

The representation like (10) holds for this function too [10]. Therefore for it there remains valid the assertion about the absence of singularities in the right half-plane $\operatorname{Re} z>0$ and of zeros on the real positive axis. Repeating the operations which have been performed for the functions $\overline{\bar{\varphi}}_{x}(p)$ and $\overline{\bar{f}}(p)$ in the domain $\mathrm{p}^{2}<0$ we are led to the following expression for $\bar{f}(p)$ which is valid in the region $0 \leqq p^{2} \leqq 4 m^{2}$

$$
\begin{aligned}
\overline{\bar{f}}(p)=-2(\pi x)^{2} \sqrt{\frac{4 m^{2}}{p^{2}}-1} \sum_{n=0}^{\infty} \frac{\partial}{\partial v} \\
\left.\cdot\left\{\frac{\left[x\left(4 m^{2}-p^{2}\right)\right]^{v}}{\Gamma(v+3) \Gamma(2 v+1)} \mathscr{P}_{-2(v+1)}^{-1}\left[\left(1-\frac{p^{2}}{4 m^{2}}\right)^{-\frac{1}{2}}\right]\right\}\right|_{v=n} .
\end{aligned}
$$

In the analytic continuation of the function $\overline{\bar{f}}(p)$ in $\mathrm{p}^{2}$ to the physical domain $p^{2}>4 m^{2}$ it is necessary to assume that the mass has a small negative imaginary part (see, e.g. Ref. [12]). Then we have

$$
4 m^{2}-p^{2}-i \varepsilon=\left(p^{2}-4 m^{2}\right) e^{-i \pi} .
$$

Using (17) as well as the formula of transformation of spherical functions [10]

$$
\begin{aligned}
& \mathscr{P}_{-2 v-2}^{-1}\left[i\left(\frac{p^{2}}{4 m^{2}}-1\right)^{-\frac{1}{2}}\right]=\frac{\left(1-\frac{4 m^{2}}{p^{2}}\right)^{\frac{1}{4}}}{\sqrt{2 \pi} \cos 2 \pi v} \\
& \left\{\sin 2 \pi v e^{-i \pi v} \Gamma(2 v+1) P_{\frac{1}{2}}^{-2 v-\frac{3}{2}}\left(2 \frac{m}{p}\right)-i \pi \frac{e^{i \pi v}}{\Gamma(2 v+3)} P_{\frac{1}{2}}^{2 v+\frac{3}{2}}\left(2 \frac{m}{p}\right)\right\},
\end{aligned}
$$

it is easy to obtain for $\overline{\bar{f}}(p)$ in the region $p^{2}>4 m^{2}$ the expression

$$
\begin{gathered}
\overline{\bar{f}}(p)=\sqrt{2 \pi}(\pi x)^{2}\left(1-\frac{4 m^{2}}{p^{2}}\right)^{\frac{3}{4}} \sum_{n=0}^{\infty}\left(i 2 \frac{\left[\varkappa\left(p^{2}-4 m^{2}\right)\right]^{n}}{(n+2) !} P_{\frac{1}{2}}^{-2 n-\frac{3}{2}}\left(2 \frac{m}{p}\right)\right. \\
\left.+\left.\frac{\partial}{\partial v}\left\{\frac{\left[\varkappa\left(p^{2}-4 m^{2}\right)\right]^{v}}{\Gamma(v+3) \Gamma(2 v+1) \Gamma(2 v+3)} P_{\frac{1}{2}}^{2 v+\frac{3}{2}}\left(2 \frac{m}{p}\right)\right\}\right|_{v=n}\right) .
\end{gathered}
$$


The formula derived are in complete agreement with the earlier ones for the massless particles. In fact, letting $m$ tend to zero we get from (19) and (13)

$$
\begin{aligned}
f_{m=0}(p) & =-(\pi \varkappa) \sum_{n=0}^{\infty} \\
& {\left[\left.\frac{\partial}{\partial v}\left\{\frac{\left[\frac{x}{4}\left(p^{2}+i \varepsilon\right)\right]^{v}}{\Gamma(v+1) \Gamma(v+2) \Gamma(v+3)}\right\}\right|_{v=n}-i \pi \frac{\left(\frac{x}{4} p^{2}\right)^{n}}{n !(n+1) !(n+2) !}\right] . }
\end{aligned}
$$

We obtained this formula earlier in the model of the neutral pion-nucleon interaction with pseudovector coupling. It entirely satisfies the requirements on the local field theory with unitary S-matrix $[2,4,13-15]$.

\section{More Strict Approximation}

After the qualitative consideration of the problem of the Fourier transformation of the function $\tilde{F}_{0}(x)$ performed in the previous section, more strict approach to this problem with the use of the approximation (5) becomes much simpler. In this approximation the integral (4) reads

$\bar{\varphi}_{x}(p)=(2 \pi x)^{2} \frac{m}{|p|} \int_{0}^{1} d t(1-t) \int_{0}^{\infty} d r r^{-2} e^{-2 r} J_{1}\left(\frac{|p|}{m} r\right) \exp \left\{-t \varkappa m^{2} \frac{e^{-r}}{r^{2}}\right\}$.

Anew we use for the exponential the Melin-Barnes integral representation. Integrating then over $t$ and changing the order of integration over $r$ and $\mathrm{z}$ we write (21) in the form

$\bar{\varphi}_{x}(p)=(2 \pi x)^{2} \frac{m}{|p|} \frac{1}{2 i} \int_{-\alpha+i \infty}^{-\alpha-i \infty} d z \frac{\left(\varkappa m^{2}\right)^{z}}{\sin \pi z \Gamma(z+3)} \int_{0}^{\infty} d r r^{-2(z+1)} e^{-r(2+z)} J_{1}\left(\frac{|p|}{m} r\right)$.

For the $z$-values in the domain $-2<\operatorname{Re} z<0$ the integral over $r$ converges and equals

$$
\begin{aligned}
& -\frac{\pi}{2} \frac{|p|}{m}(2+z)^{2 z} \frac{\left[1-\frac{p^{2}}{m^{2}(2+z)^{2}}\right]^{2 z+\frac{3}{2}}}{\sin 2 \pi z \Gamma(2 z+1)} F\left(z+\frac{3}{2}, z+2 ; 2 ; \frac{p^{2}}{m^{2}(2+z)^{2}}\right) \\
& =-\pi \frac{|p|}{m} \frac{\left[(2+z)^{2}-\frac{p^{2}}{m^{2}}\right]^{z}}{\sin 2 \pi z \Gamma(2 z+1)} \sqrt{1-(2+z)^{2} \frac{m^{2}}{p^{2}}} P_{-2 z-2}^{-1}\left[\left(1-\frac{p^{2}}{m^{2}(2+z)^{2}}\right)^{-\frac{1}{2}}\right] .
\end{aligned}
$$


It is seen from this expression that all the assertions which were valid when integrating over $\mathrm{z}$ in the function $\overline{\bar{\varphi}}_{x}(p)$ hold for the function $\bar{\varphi}_{x}(p)$ as well. Using Eq. (12) we return to the function $\bar{f}(p)$ and for the unphysical domain $\mathrm{p}^{2}<0$ we get

$\bar{f}(p)=-2(\pi x)^{2} \sum_{n=0}^{\infty} \frac{\partial}{\partial v}$

$\left.\left\{\sqrt{1-(2+v)^{2} \frac{m^{2}}{p^{2}}} \frac{\left[x\left(m^{2}(2+v)^{2}-p^{2}\right)\right]^{v}}{\Gamma(v+3) \Gamma(2 v+1)} P_{-2 v-2}^{-1}\left[\left(1-\frac{p^{2}}{m^{2}(2+v)^{2}}\right)^{-\frac{1}{2}}\right]\right\}\right|_{v=n}$.

In the next unphysical domain $0 \leqq p^{2} \leqq 4 \mathrm{~m}^{2}$ repeating the operations described in the foregoing section we obtain

$\bar{f}(p)=-2(\pi x)^{2} \sum_{n=0}^{\infty} \frac{\partial}{\partial v}$

$\left.\left\{\sqrt{(2+v)^{2} \frac{m^{2}}{p^{2}}-1} \frac{\left[x\left(m^{2}(2+v)^{2}-p^{2}\right)\right]^{v}}{\Gamma(v+3) \Gamma(2 v+1)} \mathscr{P}_{-2 v-2}^{-1}\left[\left(1-\frac{p^{2}}{m^{2}(2+v)^{2}}\right)^{-\frac{1}{2}}\right]\right\}\right|_{v=n}$.

Finally in the physical domain $p^{2} \geqq 4 m^{2}$, using formulas similar to (17) and (18) we have

$$
\begin{aligned}
\bar{f}(p)= & -2(\pi x)^{2} \sum_{n=\left[\frac{p}{m}\right]+1}^{\infty} \frac{\partial}{\partial v} \\
& \cdot\left\{\sqrt{\left.\frac{v^{2} m^{2}}{p^{2}}-1 \frac{\left[x\left(m^{2} v^{2}-p^{2}\right)\right]^{v-2}}{\Gamma(v+1) \Gamma(2 v-3)} \mathscr{P}_{-2 v+2}^{-1}\left[\left(1-\frac{p^{2}}{m^{2} v^{2}}\right)^{-\frac{1}{2}}\right]\right\}\left.\right|_{v=n}}\right. \\
+ & \sqrt{2 \pi}\left(\frac{\pi}{p^{2}}\right)^{2} \sum_{n=2}^{\left[\frac{p}{m}\right]} \frac{\partial}{\partial v} \\
& \left.\cdot\left\{\frac{\left[x\left(p^{2}-m^{2} v^{2}\right)\right]^{v}}{\Gamma(v+1) \Gamma(2 v-3) \Gamma(2 v-1)}\left(1-\frac{m^{2} v^{2}}{p^{2}}\right)^{\frac{5}{4}} P_{\frac{1}{2}}^{2 v-\frac{s}{2}}\left(\frac{m v}{p}\right)\right\}\right|_{v=n} \\
+ & i \sqrt{\frac{\pi}{2}\left(\frac{2 \pi}{p^{2}}\right)^{2}\left[\frac{p}{m}\right]} \frac{\left[x\left(p^{2}-n^{2} m^{2}\right)\right]^{n}}{n !}\left(1-\frac{m^{2} n^{2}}{p^{2}}\right)^{-\frac{5}{4}} P_{\frac{1}{2}}^{-2 n+\frac{5}{2}}\left(\frac{m n}{p}\right) .
\end{aligned}
$$

Here $\left[\frac{p}{m}\right]$ denotes the nearest integer which is smaller than $\sqrt{p^{2} / m^{2}}$. In the limit $\mathrm{m}=0$ Eqs. (24) and (26) as well as (13) and (19) reduce to Eq. (20).

When the energy tends to infinity the real part of the function $\bar{f}(p)$ tends to zero while the imaginary part increases exponentially. However, this increase does not contradict requirements imposed on local theories [13-15]. 


\section{Investigation of the Imaginary Part of the Green Function and the Meaning of the Approximation (5)}

It follows from the unitarity condition of the S-matrix that the imaginary part of the two-point Green function must be expressed in terms of the sum of the phase volumes of particles $[1,2]$

where

$$
\operatorname{Im} f(p)=8 \pi^{4} \sum_{2}^{\left[\frac{p}{m}\right]} \frac{\left(\frac{x}{4 \pi}\right)^{n}}{n !} \Omega_{n}(p),
$$

$$
\Omega_{n}(p)=\int \delta^{(4)}\left(p-\sum_{i=1}^{n} k_{i}\right) \prod_{i=1}^{n} \frac{d \boldsymbol{k}_{i}}{\omega_{i}}, \quad \omega_{i}=\sqrt{k_{i}^{2}+m^{2}} .
$$

Comparing Eqs. (26) and (27) we get in our approximation the phase volume to be equal

$\bar{\Omega}_{n}(p)=(2 \pi)^{-\frac{3}{2}} \frac{\left[4 \pi\left(p^{2}-m^{2} n^{2}\right)\right]^{n}}{\left(p^{2}\right)^{2}}\left[1-\frac{m^{2} n^{2}}{p^{2}}\right]^{-\frac{5}{4}} P_{\frac{1}{2}}^{-2 n+\frac{5}{2}}\left(\frac{m n}{p}\right)$.

It is easy to obtain from this expression the asymptotic estimates of the behaviour of the approximate phase volume at different energies. Here we give some of these estimates

$$
\bar{\Omega}_{n}(p) \approx \begin{cases}2 \pi \frac{\left(\pi p^{2}\right)^{n-2}}{(n-1) !(n-2) !}, & p \gg n m, \\ \frac{(4 \pi)^{n}(p-m n)^{2 n-\frac{5}{2}}}{(2 \pi p)^{\frac{3}{2}} \Gamma\left(2 n-\frac{3}{2}\right)}, & n \approx \frac{p}{m}, \\ \frac{(4 \pi)^{n}(p-m n)^{2 n-\frac{5}{2}}}{(4 \pi p n)^{\frac{3}{2}} \Gamma(2 n-3)}, & n \gg 1 .\end{cases}
$$

Comparing the obtained formulas with similar estimates of the behaviour of the true phase volume of n-scalar particles (see e.g. [16]) it is not difficult to see that for $p \gg n m$ the behaviour of $\bar{\Omega}_{n}(p)$ entirely coincides with that of the true phase volume and at threshold energies the former tends to zero somewhat more rapidly than the true one.

Such a behaviour of $\bar{\Omega}_{n}(p)$ is clear if our approximation is formulated in the language of the form factors. To this end let us consider the Green function of a free scalar particle in the region $x^{2}>0, x^{0}=t>0$. We choose the co-ordinate system where $x=\{t, 0\}$.

$$
\Delta^{(-)}(x)=\frac{i}{(2 \pi)^{3}} \int \frac{d \boldsymbol{k}}{2 \omega} e^{-i \omega t}=-\frac{m}{8 \pi t} H_{1}^{(2)}(m t) .
$$


The approximation (5) is equivalent to the introduction of the form factor $\frac{\omega-m}{|\boldsymbol{k}|}$ to the integrand of $\Delta^{(-)}(x)$. In fact,

$$
\bar{\Delta}^{(-)}(x)=\frac{i}{(2 \pi)^{3}} \int \frac{d \boldsymbol{k}}{2 \omega}\left(\frac{\omega-m}{|\boldsymbol{k}|}\right) e^{-i \omega t}=-i \frac{e^{-i m t}}{(2 \pi)^{2} t^{2}} .
$$

This is in agreement with the substitution (5). Therefore, the phase volume $\bar{\Omega}_{n}(p)$ can be written in the form (28), but introducing a form factor $G\left(\omega_{1}, \omega_{2}, \ldots, \omega_{n}\right)$

where

$$
\bar{\Omega}_{n}(p)=\int \delta^{(4)}\left(p-\sum_{i=1}^{n} k_{i}\right) \prod_{i=1}^{n} \frac{d \boldsymbol{k}_{i}}{\omega_{i}} G\left(\omega_{1}, \omega_{2}, \ldots, \omega_{n}\right),
$$

$$
G\left(\omega_{1}, \omega_{2}, \ldots, \omega_{n}\right)=\prod_{i=1}^{n} g\left(\omega_{i}\right), \quad g\left(\omega_{i}\right)=\sqrt{\frac{\omega_{i}-m}{\omega_{i}+m}} .
$$

At low energies the form factor $g\left(\omega_{i}\right)$ decreases as the first power of $\left|\boldsymbol{k}_{i}\right|$ and with increasing energy it tends to unity. Hence it follows

$$
\lim _{p \rightarrow \infty}\left[\bar{\Omega}_{n}(p) / \Omega_{n}(p)\right]=1 .
$$

In conclusion we give the expression for the asymptotics of the imaginary part of the Green function at high energies. To calculate it Eqs. (27) and (30 c) may be used. Replacing the sum in (27) by the integral and using the saddle point method we obtain

$$
\operatorname{Im} f(p) \sim \text { Const } p^{-3} \exp \left\{3\left(x \frac{p^{2}}{4}\right)^{\frac{1}{3}}\right\} .
$$

Such a behaviour obeys the requirements imposed on local theories [13-15].

Using Eq. (26) and the corresponding asymptotic equations for spherical functions it can be seen that the real part of the Green function decreases at large values of energy.

\section{Local Unrenormalizable Interactions (General Case)}

The general case of local and nonlocal unrenormalizable interactions of massless scalar particles was investigated in Ref. [3]. A unitary method of construction of the Green function in the momentum space was developed there. The investigations performed above allow us to generalize this method to the case of local unrenormalizable interactions of massive scalar particles. 
In the general case the two-point Green function can be written in the form of an infinite series in powers of the propagator of the free scalar particle

$$
\tilde{F}_{\mathrm{gen}}(x)=i \sum_{n=0}^{\infty} c(n)\left[-i g^{2} \Delta(x)\right]^{n},
$$

where the coefficients $c(n)$ for local interactions obey the condition [13-15]

$$
\varlimsup_{n \rightarrow \infty}|c(n)|^{\frac{1}{n}}=0 .
$$

As before we start the construction of the Fourier transform of the function (37) from a preliminary consideration of the function $\tilde{\Phi}_{\text {gen }}(x)$

$$
\tilde{\Phi}_{\mathrm{gen}}(x)=i \sum_{n=0}^{\infty} c(n)\left[i g^{2} \Delta(x)\right]^{n} .
$$

Using the approximation (5) and the intermediate regularization we go over to the momentum space

$$
\bar{\varphi}_{\varkappa}^{\mathrm{gen}}(p)=i \sum_{n=2}^{\infty} c(n) \varkappa^{n} \int d^{4} x e^{i p x}\left[\frac{\exp \left(-m \sqrt{\left.-x^{2}+i \varepsilon\right)}\right.}{x^{2}-i \varepsilon}\right]_{\mathrm{reg} .}^{n} .
$$

We consider the unphysical domain $\mathrm{p}^{2}<0$. We choose the system of co-ordinates where $p=\{0, \boldsymbol{p}\}$. Passing to the Euclidean metric and integrating over angles, we get

$$
\bar{\varphi}_{\varkappa}^{\operatorname{gen}}(p)=(2 \pi \varkappa)^{2} \frac{m}{|p|} \sum_{n=0}^{\infty}\left(-\varkappa m^{2}\right)^{n} c(n+2) \int_{l}^{\infty} d r r^{-2(n+1)} e^{-(n+2) r} J_{1}\left(\frac{|p|}{m} r\right) .
$$

Here 1 is the cutoff parameter.

Now following Ref. [3] we reconstruct the analytic function $\chi(z, r)$ which is regular in the right half-plane $\operatorname{Re} z>0$ and obeying the conditions $(\mathrm{z}=\mathrm{x}+\mathrm{iy})$
a) $|\chi(z, r)|<B e^{\Lambda|z|} \quad(\operatorname{Re} z>0, \Lambda>0, B>0)$,
b) $|\chi(i y, r)|<B e^{(\pi-\varepsilon)|y|} \quad(-\infty<y<\infty, \varepsilon>0)$

using its values $\chi(n, r)$ in a given sequence of points $\mathrm{n}=0,1,2, \ldots$

$$
\chi(n, r)=c(n+2)\left[\varkappa m^{2} \frac{e^{-r}}{r^{2}}\right]^{n} .
$$


The obtained function is written in the form

$$
\chi(z, r)=c(z+2)\left[\varkappa m^{2} \frac{e^{-r}}{r^{2}}\right]^{z}
$$

Making use of the properties (42) and (43) of this function one can find for $\bar{\varphi}_{\varkappa}^{\text {gen }}(p)$ the following integral representation

$\bar{\varphi}_{\varkappa}^{\text {gen }}(p)=-i 2(\pi \varkappa)^{2} \frac{m}{|p|} \int_{-\alpha+i \infty}^{-\alpha-i \infty} d z c(z+2) \frac{\left(\varkappa m^{2}\right)^{z}}{\sin \pi z} \int_{l}^{\infty} d r r^{-2(z+1)} e^{-(z+2) r} J_{1}\left(\frac{|p|}{m} r\right)$.

This representation is convenient by that it permits one to eliminate the intermediate regularization and to put $1=0$.

On the other hand, the conditions (42) and (43) ensure unambiguous reconstruction of the function $\chi(z, r)$ using its values $\chi(n, r)$. This is a very important feature of the transition from (41) to (46).

After omitting the intermediate regularization in the right-hand side of (46) we are led to the integral which was calculated earlier (see (23)). Now we may return to the function $\bar{f}_{\text {gen }}(p)$ in question, using anew Eq. (12). This can be done both before and after calculation of the integral over $z$. As a result we get for the unphysical domain $\mathrm{p}^{2}<0$

$$
\begin{aligned}
\bar{f}_{\mathrm{gen}}(p)= & -2(\pi x)^{2} \sum_{n=0}^{\infty} \frac{\partial}{\partial v} \\
& \cdot\left\{c(v+2) \frac{\left[x\left(m^{2}(2+v)^{2}-p^{2}\right)\right]^{v}}{\Gamma(2 v+1)}\right. \\
& \left.\cdot\left[1-(2+v)^{2} \frac{m^{2}}{p^{2}}\right]^{\frac{1}{2}} P_{-2 v-2}^{-1}\left[\left(1-\frac{p^{2}}{m^{2}(2+v)^{2}}\right)^{-\frac{1}{2}}\right]\right\}\left.\right|_{v=n} .
\end{aligned}
$$

The analytic continuation of the function $\bar{f}_{\text {gen }}(p)$ in $\mathrm{p}^{2}$ into the domain $\mathrm{p}^{2} \geqq 0$ proceeds in just the same way as that of the functions $\overline{\bar{f}}(p)$ and $\bar{f}(p)$ considered in the third and fourth sections. Therefore it is not wirth giving here these calculations.

In conclusion we indicate the conditions which should be satisfied by the coefficients $\mathrm{c}(\mathrm{n})$ so that it will be possible to reconstruct unambiguously the function $\chi(z, r)$ with the properties desired using its values $\chi(n, r)$. As is shown in Ref. [3] this condition can be written in the form

$$
\varlimsup_{n \rightarrow \infty} n^{k}|c(n)|^{\frac{1}{n}}=a(r)
$$


where $\mathrm{a}(\mathrm{r})$ is a function of $\mathrm{r}$ and $\mathrm{k}$ obeys the condition

$$
0<k \leqq 2 .
$$

Comparing the condition (48) with (38) it easily seen that the unrenormalized interactions considered here are local.

\section{Conclusion}

The investigation performed is a natural generalization of the methods suggested earlier by the author for the description of unrenormalizable interactions of massless particles $[1-4]$ to the case of massive particles. The approximation which has been used in constructing the Green function in the momentum space is the most interesting one from the point of view of the right qualitative description of the Green function behaviour on different domains of the variable $\mathrm{p}^{2}$. At the same time, at high energies this approximation gives a good quantitative description. At energies close to the threshold one we have somewhat underestimated results.

The method developed is applicable to the description of a rather wide class of local unrenormalizable interactions of elementary particles and obeys the requirements imposed on the theory with unitary S-matrix.

In conclusion the author is grateful to Prof. D. I. Blokhintsev for the interest in the work, and to Dr. G. V. Efimov for useful discussions.

\section{References}

1. Volkov, M. K.: Yader. Fiz. 6, 1100 (1967); 7, 445 (1968).

2. - Commun. Math. Phys. 7, 289 (1968).

3. - Ann. Phys. (N. Y.) 49, 202 (1968).

4. - Preprint ITF 69, 5 (1969).

5. Arnowitt, R., and S. Deser: Phys. Rev. 100, 349 (1955).

6. Okubo, S.: Nuovo Cimento 19, 574 (1961).

7. Guttınger, W., and E. Pfaffelhuber: Nuovo Cimento 52, 389 (1967).

8. Lee, T. D.: Preprint CERN Geneva 68/940/5 (1968).

9. Watson, G. N.: Theory of Bessel functions. New York: Cambridge University Press 1944.

10. Erdèlyi, A. (Ed.): Higher transcendental functions, Vol. 1. New York-TorontoLondon: McGraw-Hill 1953.

11. Gradstein, I. S., and I. M. Ryzhik: Tables of integrals, sums, and products. Moscow: GIFML 1962.

12. Bogolubov, N. N., and D. V. Shirkov: Introduction to the theory of quantized fields. New York: Addison-Wesley 1959.

6 Commun math Phys, Vol 15 
13. Meiman, N. N.: JETP 47, 1966 (1964).

14. Jaffe, A. M.: Ann. Phys. (N. Y.) 32, 127 (1965); Phys. Rev. Letters 17, 661 (1966).

15. Efimov, G. V.: Commun. Math. Phys. 7, 138 (1968).

16. Kolkunov, V. A.: JETP 43, 1448 (1962).

17. Evgrafov, M. A.: Asymptotic estimates and entire functions. Moscow: GIFML 1962.

M. K. Volkov

Joint Institute for Nuclear Research

Head Post Office P. O. Box 79

Moscow, USSR 